\title{
Uma Iniciativa para Aumentar a Participação Feminina nas Competições de Programação: um Relato de Experiência
}

\author{
Alice Lima $^{1}$, Maria Eduarda Santos ${ }^{1}$, \\ Tong Zhou ${ }^{1}$, Maristela Holanda ${ }^{1}$, Aleteia P. F. Araujo ${ }^{1}$, \\ Carla Cavalcante Koike ${ }^{1}$, Vinícius R. P. Borges ${ }^{1}$, Roberta B. Oliveira ${ }^{1}$ \\ ${ }^{1}$ Departamento de Ciência da Computação (CIC) - Universidade de Brasília (UnB) \\ Brasília, DF - Brasil \\ \{alice.lima, carvalho.maria, tong.zhou\}@aluno.unb.br, \\ \{mholanda, aleteia, ckoike, viniciusrpb, roberta.oliveira\}@unb.br
}

\begin{abstract}
Programming competitions may provide several skills to participants. Currently, female participation is still not very significant in programming competitions. This paper presents an initiative carried out in the Department of Computer Science of the University of Brasilia, with the objective of increasing the number of girls in programming competitions and therefore, allowing them to have opportunities to obtain the skills provided by the competitions. The results of the initiative showed that girls felt more motivated and they participated in more activities related to programming competitions than in previous years.
\end{abstract}

Resumo. Competições de programação podem proporcionar várias habilidades aos participantes. Atualmente, a participação feminina ainda não é muito expressiva em competições de programação. O presente artigo apresenta uma iniciativa realizada no Departamento de Ciência da Computação da Universidade de Brasília, com o objetivo de aumentar o número de meninas nas competições de programação e, portanto, possibilitar que elas tenham oportunidades para adquirir as habilidades proporcionadas pelas competições. Os resultados da iniciativa mostraram que as meninas sentiram-se mais motivadas e participaram de mais atividades relacionadas a competições de programação do que em anos anteriores.

\section{Introdução}

A participação feminina em competições de programação, embora venha apresentando números crescentes, ainda é uma porcentagem pequena quando comparada a participação geral, masculina e feminina. Isso se deve, em grande parte, por ser uma área em que a presença masculina é historicamente mais predominante, como é discutido em [Pessoa et al. 2017a] . No entanto, há diversas iniciativas com o propósito de mudar esse cenário. Essas iniciativas têm como objetivos: (1) aumentar a participação feminina em competições de programação [Freitas et al. 2016b, Pessoa et al. 2017a, Bastos et al. 2017]; (2) atrair meninas para os cursos de computação [Molnar et al. 2021, Maciel and Bim 2016]; e (3) evitar a evasão de meninas iniciantes em programação [Franzoia et al. 2019].

Vários projetos apresentam resultados promissores com a realização de atividades para competições de programação, não somente com o aumento da participação 
feminina [Freitas et al. 2016a, Pessoa et al. 2017b], mas também com o aumento da participação geral nas competições [Piekarski et al. 2015]. Por exemplo, o trabalho de [Freitas et al. 2016b] teve como objetivo incentivar as estudantes a aumentar suas habilidades de programação. Os autores propuseram como metodologia o desenvolvimento de aplicativos móveis e a participação feminina em competições de programação. Os resultados apresentaram aumento na influência feminina nos cursos de Computação e os aplicativos desenvolvidos estiveram presentes na mídia.

Com o objetivo de aumentar o interesse dos(as) alunos(as) nas maratonas de programação, [Pessoa et al. 2017a] propuseram uma metodologia para a inclusão de alunas por meio de palestras e o treinamento destas por meio de atividades de programação. Com o mesmo objetivo, [Bastos et al. 2017] propuseram uma metodologia dividida em quatro partes: obter a atenção das alunas em programação, treinar as habilidades de programação dessas meninas, desenvolver suas habilidades de programação, e finalmente, identificar as deficiências individuais de cada uma. Além disso, com a metodologia proposta foi possível incentivar outras alunas a participarem e fomentar novos desafios a serem enfrentados.

A participação feminina em competições de programação sempre foi incentivada pelo grupo de maratonas do Departamento de Ciência da Computação (CIC) da Universidade de Brasília (UnB). No entanto, poucos resultados tinham sido obtidos antes da realização do presente trabalho. Em 2020 havia pouca participação feminina na maioria das competições de programação. Considerando esse problema, uma iniciativa foi realizada para aumentar o número de meninas do CIC nas competições de programação. Além de aumentar a representatividade feminina, a participação em maratonas pode fornecer várias habilidades às mesmas, como o aumento da aptidão em resolução de problemas, o estímulo à criatividade e a capacidade do trabalho em equipe [Piekarski et al. 2015, Paganini and Gama 2020]. O objetivo do presente artigo é apresentar as atividades que foram desenvolvidas no primeiro semestre letivo de 2020 no CIC de forma remota, devido à situação de pandemia de SARS-CoV-2. Além disso, são analisados os resultados a partir da realização das atividades, bem como a elaboração de estratégias que serão adotadas em atividades futuras para dar continuidade a iniciativa.

O presente artigo contém a seguinte estrutura: Na Seção 2 é feita um breve panorama da participação feminina nas competições de programação. Na Seção 3 são apresentadas as atividades realizadas pelo grupo em seu primeiro semestre de existência. $\mathrm{Na}$ Seção 4 são discutidos os resultados que foram obtidos até o momento. Na Seção 5 são abordadas as estratégias para atividades futuras que serão adotadas, com o intuito de contribuir para uma participação maior de meninas em competições de programação. Na Seção 6 são apontadas as conclusões em relação às atividades desenvolvidas.

\section{Histórico de Participação Feminina do CIC/UnB em Competições de Programação}

$\mathrm{O}$ grupo de maratonas de programação do $\mathrm{CIC} / \mathrm{UnB}^{1}$ organiza várias maratonas de programação de âmbito no Distrito Federal (DF), como por exemplo, Maratona UnB, Seletiva UnB e Maratona Instituto Federal de Brasília (IFB). A Maratona UnB e a Seletiva

\footnotetext{
${ }^{1}$ http://maratona.cic.unb.br/
} 
UnB para a fase regional da Maratona SBC de Programação ${ }^{2}$ foram realizadas de maneira presencial em 2018 e 2019, e de maneira remota em 2020. Na Tabela 1 pode ser visto o número de meninas participantes de tais competições em relação aos inscritos do CIC nos últimos 3 anos. Em 2018, 1 aluna do CIC participou da Maratona UnB (local), e nenhuma aluna participou da Seletiva UnB (local). Em 2019, apesar do número de participantes do CIC inscritos na Maratona UnB aumentar, apenas 1 aluna participou da competição. No caso da Seletiva UnB, o cenário foi o mesmo do ano anterior. Já em 2020, a presença feminina aumentou, considerando a participação de 6 meninas do CIC na Maratona UnB e a participação de 7 meninas na Seletiva UnB. A percepção sobre esse aumento será apresentada na Seção 4.

Tabela 1. Participação de meninas em competições de programação em relação aos inscritos do CIC nos anos de 2018 a 2020.

\begin{tabular}{|c|c|c|c|c|c|c|c|c|}
\hline & \multicolumn{2}{|c|}{ OBI (Fase 1) } & \multicolumn{2}{|c|}{ Maratona UnB } & \multicolumn{2}{|c|}{ Seletiva UnB } & \multicolumn{2}{|c|}{$\begin{array}{l}\text { Maratona SBC } \\
\text { (Fase Regional) }\end{array}$} \\
\hline & Meninas & Inscritos & Meninas & Inscritos & Meninas & Inscritos & Meninas & Inscritos \\
\hline 2018 & 2 & 7 & 1 & 17 & 0 & 15 & 0 & 12 \\
\hline 2019 & 7 & 53 & 1 & 39 & 0 & 15 & 0 & 15 \\
\hline 2020 & 3 & 26 & 6 & 34 & 7 & 58 & 11 & 57 \\
\hline
\end{tabular}

O primeiro registro de participação do CIC em maratonas oficiais de programação da SBC, considerando a Fase Regional (primeira fase), foi no ano de 1996. Para algumas competições na Fase Regional, não foi possível obter as informações dos integrantes dos times participantes pelos responsáveis atuais, portanto, não foi identificado se houve a presença de meninas nos times que participaram dessas competições. Há registros de participação de uma menina do CIC em um time no ano de 2009 (de 5 times inscritos), e outra menina no único time do CIC inscrito no ano de 2013. A participação de meninas do CIC em maratonas oficiais da SBC foi mais expressiva em 2020, comparada aos anos anteriores de 2018 e 2019, que não teve participação feminina (Tabela 1). Ao contrário dos outros anos, a Fase Regional de 2020 foi online e cada instituição pôde inscrever vários times. Somente os times que participaram e que cumpriram os requisitos da Seletiva UnB (acertar 3 ou mais questões) poderiam inscrever-se na Fase Regional da Maratona SBC representando o CIC, com exceção de vagas adicionais de inclusão, por exemplo para a participação feminina. O CIC foi representado por 19 times (57 participantes), sendo 2 times femininos, 13 times masculinos e 4 times compostos por meninos e meninas. Portanto, ao todo foram 11 meninas do CIC que participaram desta competição. Uma análise sobre a participação feminina será apresentada na Seção 4.

No caso das competições da Olimpíada Brasileira de Informática (OBI) $)^{3}$ o CIC é apenas uma das sedes no DF e no Brasil. Entretanto, o CIC é responsável pelas inscrições e pela organização do local de prova, que geralmente considera os níveis 2 e sênior da modalidade de programação. O nível 2 engloba alunos do ensino fundamental e de alunos até o terceiro ano do ensino médio, e o nível sênior corresponde aos alunos do quarto ano de escolas técnicas ou alunos que estão cursando, pela primeira vez, o primeiro ano de um curso de graduação. Considerando a Fase 1 (local) da OBI dos três últimos anos do nível sênior (Tabela 1), as competições foram presenciais, com exceção do ano de 2020,

\footnotetext{
${ }^{2} \mathrm{http}: / /$ maratona.sbc.org.br/

${ }^{3}$ https://olimpiada.ic.unicamp.br/
} 
que ocorreu de forma remota, devido ao cenário de pandemia. Neste contexto, 2 alunas do CIC participaram no ano de 2018. Em 2019, houve um aumento de alunas inscritas na OBI, sendo 7 alunas do CIC. No entanto, o número total de participantes foi bem maior do que o ano anterior. No caso de 2020, apenas 3 meninas participaram na Fase 1, diminuindo o número de interessadas na competição em relação ao ano anterior, mas o número de inscritos também foi menor. Uma comparação da participação de meninas do CIC em algumas competições nos últimos 3 anos pode ser vista na Figura 1 .

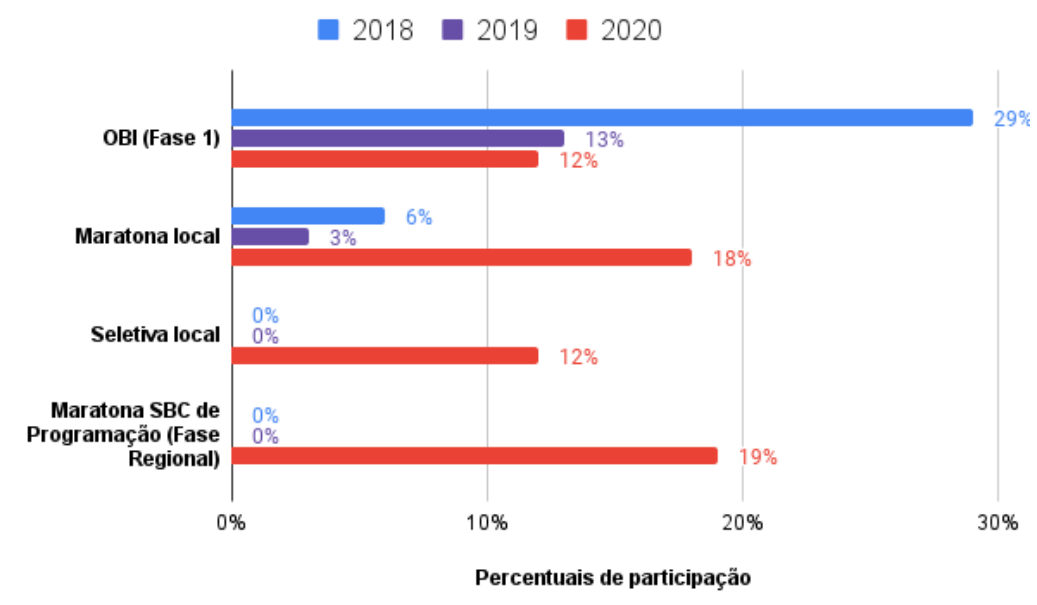

Figura 1. Participação feminina do CIC em competições de programação.

\section{Atividades Realizadas}

Com o intuito de aumentar a participação feminina nas competições de programação, algumas atividades foram realizadas no primeiro semestre letivo de 2020 para motivar as meninas vinculadas ao CIC, tais como: 1) criação e divulgação de um grupo online de interação e treinamento, 2) mensagens de incentivos para treinamento de programação competitiva e formação de equipes femininas do CIC, 3) divulgação de competições de programação e eventos com foco em meninas, e, principalmente, 4) criação de um grupo de treinos específicos para as meninas.

O grupo online de interação e treinamento para as meninas foi criado no início do primeiro semestre de 2020, na qual o aplicativo Telegram ${ }^{4}$ foi usado para a comunicação entre as integrantes do grupo. O grupo faz parte do projeto de extensão Meninas.comp $\mathrm{f}^{5}$ e tem parceria com o grupo de maratonas de programação, ambos vinculados ao CIC. O grupo foi formado por 16 meninas, que compõem os cursos de Ciência da Computação, Licenciatura em Informática e Engenharia Mecatrônica. Algumas meninas, que já demostravam interesse em programação competitiva de acordo com a indicação de alguns professores, foram convidadas para o grupo e outras souberam do grupo por indicação de algum amigo ou divulgação em grupos de aplicativos de troca de mensagens.

As mensagens de incentivo para treinamento eram enviadas pelos professores responsáveis ou pelas próprias integrantes do grupo, tais como, a indicação da disciplina de programação competitiva oferecida pelo CIC e de atividades de outros projetos da $\mathrm{UnB}$, por exemplo o projeto BOSS6 As meninas foram incentivadas a participar de cur-

\footnotetext{
${ }^{4}$ https://telegram.org/

${ }^{5}$ https://www.meninas.cic.unb.br/

${ }^{6}$ https://github.com/BOSS-BigOpenSourceSibling/BigSibling
} 
tas simulações de maratonas de programação, com o objetivo de programarem algoritmos em um tempo estabelecido para problemas que serão testados, conforme disponibilizados na plataforma Codeforces ${ }^{7}$. Além disso, também foram incentivadas a formar equipes femininas para os treinamentos e competições. Vários eventos foram divulgados no grupo com o intuito de aproximar as meninas das temáticas sobre competições ou meninas na programação.

Encontros online foram realizados com objetivo de proporcionar interação entre as integrantes do grupo e identificar as suas necessidades. A partir dessas reuniões foi possível notar o interesse delas em treinamentos específicos para meninas. Portanto, foi criado um grupo de treinos com colaboração de alguns integrantes do grupo da UnBalloon da UnB. Inicialmente, os colaboradores do grupo de treino consideraram alguns problemas de competições de programação com o objetivo de identificar o nível de programação das meninas, e em seguida disponibilizaram exercícios para serem resolvidos individualmente e também em equipe. Reuniões online foram propostas quando necessário para esclarecer dúvidas, discutir soluções e apresentar estratégias, além da comunicação por mensagens.

\section{Resultados da Realização das Atividades}

Com o objetivo de analisar os resultados obtidos a partir da realização das atividades no grupo, foi solicitada a participação das meninas do grupo na pesquisa por meio de um formulário de percepção. O formulário de percepção foi disponibilizado na configuração online e teve sua divulgação no grupo do Telegram. Este é composto por 31 questões divididas em cinco seções. A primeira seção do formulário coletou os dados pessoais, como a idade das alunas. A segunda seção coletou as informações acadêmicas (curso, período e experiência com programação). A terceira seção foi composta de perguntas sobre o interesse e a participação em competições de programação. A quarta seção abordou questões sobre atividades de treino para competições de programação e, por último, a quinta seção foi composta por questões a respeito do grupo criado.

É importante ressaltar que o grupo contava com 16 meninas em 2020, porém apenas 13 participaram da pesquisa. Destas, 61,53\% cursam Ciência da Computação, 15,38\% cursam Licenciatura em Informática e 23,07\% cursam Engenharia Mecatrônica. Percebese a ausência de alunas do curso de Engenharia de Computação no grupo, sendo que este também está vinculado ao CIC. No primeiro semestre de 2020, o número de alunas matriculadas em todos os cursos de computação mencionados anteriormente totalizava 200. Portanto, é possível identificar que poucas meninas do departamento estão envolvidas com as atividades relacionadas a competições de programação.

Em relação ao período de curso das integrantes do grupo, $15,4 \%$ das alunas estão no primeiro ano, $38,5 \%$ estão no segundo ano, $7,7 \%$ estão no terceiro, $30,8 \%$ estão no quarto e 7,7\% estão no sexto ano. É possível observar que a maioria se encontra no segundo ou quarto ano de curso. Além disso, é importante atentar-se à baixa participação de meninas que estão no primeiro ano de curso, uma vez que é nesse período que são cursadas as disciplinas introdutórias de programação e estruturas de dados, que são importantes para as competições de programação. Uma outra questão que deve ser investigada é a baixa participação de estudantes do terceiro ano, uma vez que a presença de meninas no ano seguinte é consideravelmente superior.

\footnotetext{
${ }^{7}$ https://codeforces.com/
} 


\section{Experiência das participantes}

Um aspecto importante para compreender melhor o perfil das participantes do grupo é a experiência com programação, portanto, foi perguntado se elas possuíam facilidade em programar, isto é, se conseguem desenvolver um programa a partir de um problema proposto utilizando os elementos básicos de programação.

Como mostrado na Figura 2, a maioria das meninas considera que tem facilidade em programação (61,5\% das participantes). Além disso, foi constatado que 46,2\% das participantes estavam cursando no semestre atual alguma disciplina de programação competitiva da universidade, tendo a maioria, 53,8\%, não cursado até o momento. Geralmente, nas disciplinas de programação competitiva são apresentadas técnicas de programação para as competições e são realizados simulados de competições em plataformas próprias para esse tipo de atividade, com o objetivo de contribuir com conhecimento e experiência para as competições.

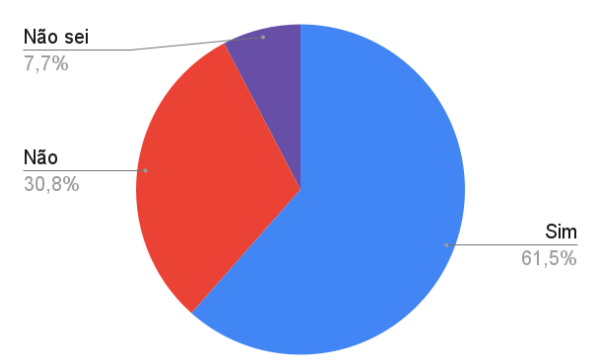

Figura 2. "Você considera
que tem facilidade em
programação?"
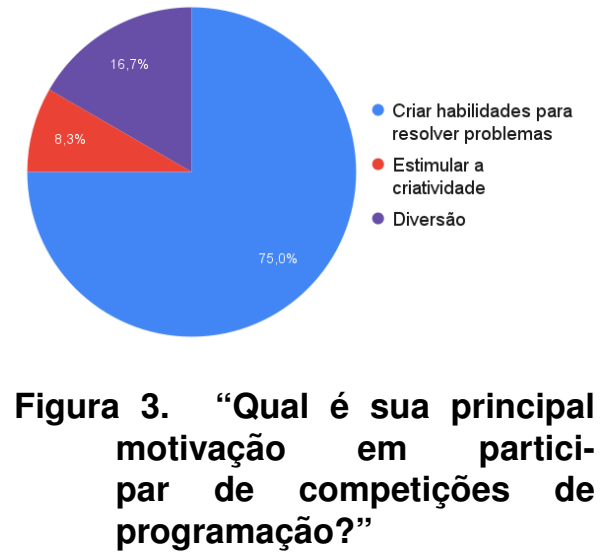

\section{Envolvimento nas competições de programação}

Outra questão analisada foi a principal motivação das meninas que tinham interesse em participar de competições de programação (92,3\% de interessadas). Assim, conforme indicado na Figura 3, 75\% das meninas apontaram criar habilidades para solucionar problemas como principal motivação em participar de competições, 8,3\% estimular a criatividade e $16,7 \%$ diversão. Nenhuma aluna considerou a motivação de desenvolver a capacidade de trabalhar em equipe ou apontou qualquer outra motivação. Todas as opções são habilidades que podem ser obtidas com a participação em competições, conforme apresentadas em [Piekarski et al. 2015, Paganini and Gama 2020]. A única aluna que não tinha interesse em participar de competições naquele período, justificou informando que não possuía tempo para os treinamentos. Além disso, 46,2\% das meninas informaram já ter participado de alguma competição antes do primeiro semestre de 2020.

\section{Percepção sobre as atividades do grupo}

A última seção do questionário procurou investigar a percepção das meninas em relação ao grupo, com a pergunta aberta "Qual é o principal objetivo em participar do grupo de meninas com foco em maratonas de programação?", como resposta, os principais objetivos apontados pelas meninas foram: aprendizado, melhoria das habilidades, acolhimento, diversão e engajamento. A fim de descobrir a contribuição do grupo para as alunas, algumas perguntas sobre esse aspecto também foram consideradas, conforme 
apresentadas na Figura 4. A partir da análise dos resultados apresentados na Figura 4(a) é possível concluir que para a maioria $(84,6 \%)$ o grupo trouxe motivação para participar das atividades relacionadas à maratonas de programação. Devido ao incentivo do grupo, 69,2\% participaram de algum treinamento (Figura 4(b)), e 61,5\% participaram de alguma competição (Figura 4(c)). Para 84,6\% das alunas, a percepção em relação às maratonas de programação mudou após a participação no grupo, conforme apresentado na Figura 4(d). Ao decorrer do primeiro semestre da existência do grupo, as alunas participaram das seguintes competições: (1) Olimpíada Brasileira de Informática ${ }^{8}$, primeira fase do nível sênior da modalidade de programação; (2) XXV Maratona SBC de Programação 9 , primeira fase; (3) WIT - Maratona Day, realizada durante o evento CSBC 2020 - Escola de Inverno ${ }^{10}$; (4) Seletiva UnB ${ }^{11}$, para a Fase Regional da Maratona SBC de Programação; e (5) Maratona $\mathrm{UnB}^{11}$, realizada na UnB.

(a)

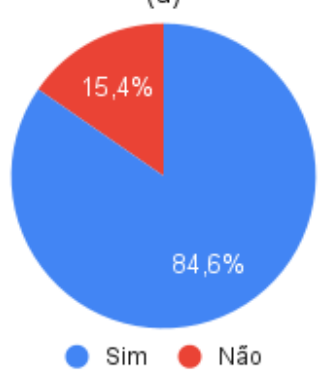

(c)

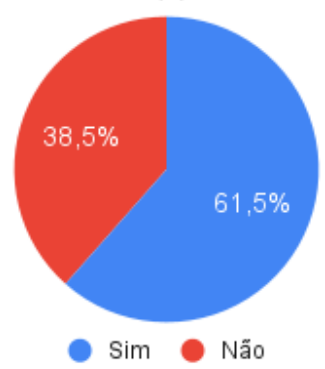

(b)

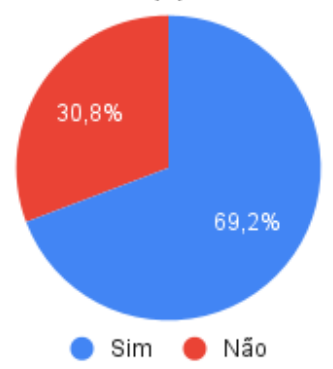

(d)

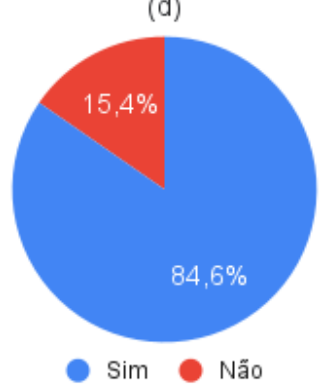

Figura 4. Perguntas a respeito da contribuição do grupo para a participação das alunas em atividades de programação competitiva: (a) "Com o grupo você se sentiu mais motivada em participar de eventos/treinamentos/competições relacionados a maratonas de programação?", (b) "Você participou de algum treinamento devido ao incentivo no grupo?", (c) "Você participou de alguma competição devido ao incentivo no grupo?”, (d) "A sua percepção em relação as maratonas de programação mudou após a participação no grupo?”

É importante salientar que a OBI, na modalidade mencionada anteriormente, só admite participantes de nível superior que estejam cursando o primeiro ano, dessa forma, nem todas as integrantes do grupo puderam se inscrever. No caso na maratona SBC,

\footnotetext{
${ }^{8}$ https://olimpiada.ic.unicamp.br/

${ }^{9} \mathrm{http}: / /$ maratona.sbc.org.br/

${ }^{10} \mathrm{http}: / /$ www.sbc.org.br/csbc2020/

${ }^{11} \mathrm{http}: / /$ maratona.cic.unb.br/unb
} 
também tem algumas restrições para a inscrição, que eram os casos de algumas meninas do grupo. Sobre a ausência nos treinamentos ou competições, a maioria das meninas respondeu que o motivo de não participar foi a falta de tempo. Considerando as meninas que participaram de alguma competição, 87,5\% consideraram que as atividades contribuíram para melhorar o desempenho nas competições.

No ano de 2020, participaram da Primeira Fase da Maratona SBC de Programação 19 equipes do departamento, compostas por três integrantes cada, totalizando 57 participantes. Deste total, 11 participantes do departamento eram do gênero feminino, sendo que 2 equipes eram totalmente femininas e 4 mistas, isto é, composta por meninas e meninos, conforme discutido na Seção 2. Os times femininos apresentaram um ótimo desempenho, assim como as meninas participantes dos outros times. Considerando as 11 meninas que participaram, 10 eram integrantes do grupo de acordo com os registros. Esse cenário foi diferente nas maratonas dos anos de 2018 e 2019, que não houve registros de participação feminina em tal evento. Também é importante salientar que na edição de 2020, o número de equipes por instituição não era limitado como nos anos anteriores. A respeito da participação nas maratonas locais, como mostrado na Figura 1, enquanto o número de participação feminina na OBI diminuiu, nas demais competições locais houve um aumento significativo de meninas interessadas.

\section{Estratégias Propostas para Atividades Futuras}

Apesar dos resultados promissores obtidos com a realização das atividades apresentadas na Seção 4, é possível observar que a participação das meninas em maratonas de programação ainda é baixa, uma vez que poucas alunas do CIC/UnB interessaramse pelas competições em comparação a participação masculina. De acordo com [Varol and Varol 2014], é importante ter um ambiente confortável para as alunas, o qual torne a prática de preparos para competições viável. Portanto, com o objetivo de dar continuidade no oferecimento de um ambiente mais confortável para que as meninas sintam-se mais motivadas para aprender e desenvolver habilidades para as competições, pretende-se considerar mais atividades com foco em treinamento. Com essas atividades, espera-se que as meninas sintam-se mais confiantes e preparadas para integrar o grupo geral da UnB, no qual elas terão oportunidades de adquirir mais conhecimento e interação.

Com o objetivo de aumentar a interação entre as interessadas no assunto, pretendese considerar atividades como o desenvolvimento de soluções computacionais em duplas e palestras motivacionais sobre maratonas de programação [Alvarado et al. 2012]. A elaboração de soluções computacionais envolvendo dois discentes é um método utilizado por docentes para aumentar o desempenho dos alunos nas disciplinas introdutórias de programação [Wang et al. 2019]. Portanto, esse tipo de atividade pode também ajudar a aumentar a participação de alunas do primeiro ano em competições, como a OBI, que conforme apresentado na Seção 4, registrou baixa participação. Palestras com treinadores, competidores, ex-competidores e especialistas podem ajudar na motivação das alunas e melhor gerenciamento do tempo, uma vez que esse foi um fator predominante que impediu a participação de algumas meninas nos treinamentos e, consequentemente, nas competições.

Para maximizar o desempenho das atuais interessadas nas competições e minimizar a evasão das mesmas, pequenos simulados podem ser semanalmente aplicados, compondo-se de questões de implementação livre, as quais poderão posteriormente ser 
discutidas em seminários de soluções eficientes dos problemas, normalmente utilizadas em competições, para que, então, haja a aplicação dos métodos compreendidos em horário de simulação. Esta abordagem se mostra eficiente em diversos casos de estudo [Alvarado et al. 2012], e tem como objetivo estimular a busca por soluções dinâmicas por parte das integrantes do grupo, já que a apresentação das soluções eficientes serão ministradas pelas mesmas, em reuniões organizadas pelos responsáveis.

Por fim, para que o aumento do interesse por maratonas de programação não se restrinja apenas às alunas de um determinado período dos cursos de graduação, faz-se necessário reservar parte das atividades propostas para divulgação e ampliação das integrantes do grupo de estudos. Com o propósito de buscar mais participantes para as competições, as mídias sociais serão utilizadas para a difusão do projeto no departamento, em outras instituições, assim como em eventos regionais e nacionais para alcançar mais meninas. Também, o contato com outras organizações dentro do departamento, tais como empresas júniores, atlética esportiva, direção de representação dos alunos é necessário para que mais meninas possam conhecer as vantagens da participação de competições de programação e então, participar das atividades aqui propostas. Além disso, é importante aumentar o número de meninas nos cursos do departamento para que as atividades possam alcançar mais meninas, portanto, pretende-se desenvolver atividades relacionadas às competições para meninas no ensino médio, a partir de um projeto de extensão apoiado pelo Meninas.comp.

\section{Conclusão}

$\mathrm{O}$ presente artigo apresenta uma iniciativa realizada no CIC/UnB no primeiro semestre letivo de 2020, com o objetivo de aumentar o número de meninas nas competições de programação. Para alcançar esse objetivo, foi criado um grupo especificamente de meninas, e algumas atividades foram realizadas. O grupo era composto por 16 meninas de cursos vinculados ao departamento. Apesar do número ser baixo em relação ao total de matrículas, é um número razoável considerando o número de interessadas no departamento, atualmente. Os resultados obtidos a partir da realização das atividades mostraram-se promissores, pois o número de meninas que participaram de competições de programação em relação aos anos anteriores foi maior, com exceção da OBI. No entanto, o grupo não contava com muitas integrantes do primeiro ano, conforme as restrições da competição. Além disso, a maioria das participantes relataram que a participação nos treinamentos e competições ocorreram devido aos incentivos do grupo.

Mesmo com os resultados promissores apresentados pelas atividades desenvolvidas, a participação feminina ainda é pequena quando comparada à participação masculina. Isso pode ser justificado pela grande maioria de meninos matriculados nos cursos vinculados ao departamento. Então, faz-se necessário desenvolver e divulgar mais as atividades do grupo, com o intuito de atrair mais meninas. Outro problema a ser considerado é realizar atividades com o objetivo de aumentar o número de meninas matriculadas nos cursos de computação com interesse em competições de programação. Além disso, outras atividades com foco em um treinamento estruturado para preparar as alunas para as competições de programação são necessárias para ajudar a melhorar o desempenho das mesmas nas competições, além de todos os outros benefícios que elas podem adquirir com as competições. Considerando a implementação dessas atividades, espera-se que o interesse pelo projeto aumente, e que, deste modo, as meninas do departamento possam conquistar posições de destaque nos eventos relacionados às competições de programação. 


\section{Referências}

Alvarado, C., Dodds, Z., and Libeskind-Hadas, R. (2012). Increasing women's participation in computing at harvey mudd college. ACM Inroads, 3(4):55-64.

Bastos, M. S., Tamayo, S. C., and Franco, E. M. (2017). Motivacion y autoeficacia en mujeres que estudian carreras de perfil informático. In Congreso de la Mujer Latinoamericana en Computación (LAWCC-CLEI)-JAIIO 46 (Córdoba, 2017).

Franzoia, F., Pires, F., and Pessoa, M. (2019). Mentorando meninas iniciantes em programação: um estudo de caso. In Anais do XIII Women in Information Technology, pages 199-203. SBC.

Freitas, R., Lobo, L., Aires, V. P., Dantas, N., and Conte, T. (2016a). Programming contests and mobile apps development as actions for attracting and retaining brazilian women in computing courses. In VIII Latin American Women in Computing Congress (LAWCC), CLEI.

Freitas, R., Lobo, L., and Conte, T. (2016b). Projeto scitechgirls: desenvolvimento de aplicativos e participação em competições de programação científicas e tecnológicas. In Anais do X Women in Information Technology, pages 87-91. SBC.

Maciel, C. and Bim, S. A. (2016). Programa meninas digitais-ações para divulgar a computação para meninas do ensino médio. Anais do Computer on the Beach, pages 327-336.

Molnar, A., Keane, T., and Stockdale, R. (2021). Educational interventions and female enrollment in it degrees. Communications of the ACM, 64(3):73-77.

Paganini, L. and Gama, K. (2020). Engaging women's participation in hackathons: A qualitative study with participants of a female-focused hackathon. In International Conference on Game Jams, Hackathons and Game Creation Events 2020, ICGJ20, page 8-15, New York, NY, USA. Association for Computing Machinery.

Pessoa, M. S. P., Tamayo, S. C., and Franco, E. M. (2017a). Panorama da participação feminina em competições de programação promovida por instituição de ensino superior da região norte. In Anais do XI Women in Information Technology. SBC.

Pessoa, M. S. P., Tamayo, S. C., and Rodrigues, J. (2017b). Aumento da participação feminina em competições de programação tecnológicas através da criação de grupos de treinamento e pesquisa. In Anais do XI Women in Information Technology. SBC.

Piekarski, A. E., Miazaki, M., Hild, T., Mulati, M. H., and Kikuti, D. (2015). A metodologia das maratonas de programação em um projeto de extensão: um relato de experiência. In Anais dos Workshops do Congresso Brasileiro de Informática na Educação, volume 4, page 1246.

Varol, H. and Varol, C. (2014). Improving female student retention in computer science during the first programming course. International Journal of Information and Education Technology, 4(5):394.

Wang, S., Andrei, S., Urbina, O., and Sisk, D. A. (2019). A coding/programming academy for 6th-grade females to increase knowledge and interest in computer science. In 2019 IEEE Frontiers in Education Conference (FIE), pages 1-8. IEEE. 